http://dx.doi.org/ 10.22319/rmcp.v9i1.4376

Artículo

\title{
Valoración contingente y disponibilidad a pagar por atributos intangibles en carne de bovino
}

\section{Contingent valuation and willingness to pay for credence attributes in beef meat}

José Luis Jaramillo Villanueva ${ }^{\mathrm{a}^{*} \text {, }}$

Samuel Vargas López, ${ }^{\mathrm{a}}$

Lissete Abigail Rojas Juárez ${ }^{\mathrm{a}}$

${ }^{\text {a }}$ Colegio de Posgraduados, Campus Puebla. Boulevard Forjadores 205, Momoxpan, San Pedro Cholula, Puebla. C.P. 72760.

*Autor para correspondencia. jaramillo@ colpos.mx

\section{- Resumen:}

La demanda de carne de res disminuyó en los últimos años, por lo que analizar la estructura de preferencia de los consumidores es importante. La presente investigación utilizó el Método de valoración contingente (MVC) para determinar las preferencias de los consumidores por los atributos inocuidad, orgánico, precio, contenido de grasa, frescura y color, para descubrir su nicho de mercado. A partir de datos de encuesta a una muestra de 225 hogares, en ciudades del estado de Puebla, se realizó análisis de correlación y un modelo econométrico Probit, para identificar las variables explicativas de la Disponibilidad a pagar (DAP) un sobreprecio por los atributos inocuidad y orgánico. El consumo per cápita de carne fue de $19.4 \mathrm{~kg} /$ año, con un coeficiente de variación de 0.205 . El $80 \%$ de los consumidores creen que la carne que compran puede estar contaminada en algún grado con sustancias o microorganismos que pueden dañar su salud. El 76 y $66 \%$ de los consumidores tienen DAP un sobreprecio por los atributos inocuidad y orgánico, respectivamente. La DAP promedio por estos atributos es de 13 y $12.1 \%$ respectivamente. Los factores explicativos y significativos $(P<0.05)$ de la DAP son la edad, la escolaridad, el ingreso, la percepción del riesgo, y los atributos contenido de grasa, frescura y suavidad. Los primeros cuatro están asociados con efectos marginales mayores al $20 \%$. El 14.4 y $19.1 \%$ de la muestra tienen DAP mayor al $25 \%$ del precio que actualmente pagan, porque su carne sea orgánica e inocua, respectivamente. 
- Palabras clave: Inocuidad, Carne orgánica, Disponibilidad a pagar, Valoración contingente, Preferencias de consumidores.

\begin{abstract}
- Abstract:
The demand for beef has declined in recent years, so it is important to know the structure of consumers' preferences. The present research used the contingent valuation method (CVM) to determine consumers' preferences for the attributes of food safety, organic, price, fat content, freshness and color, to discover their potential niche market. Based on survey data from a random sample of 225 households in eight cities of the State of Puebla, correlation analysis and an econometric Probit model were performed to identify the explanatory variables of the Willingness to Pay (WTP) a price premium for the attributes safety and organic. The per capita consumption of beef meat was $19.4 \mathrm{~kg} / \mathrm{yr}$. The $80 \%$ of consumers believe that meat they buy may be in some degree contaminated with substances or microorganisms that can cause damage to their health. The 76 and $66 \%$ of consumers have a WTP a premium for the attributes safety and organic, respectively. The average WTP for these attributes is 13 and $12.1 \%$, respectively. The explanatory factors of the WTP are age, schooling, income, risk perception, and the attributes fat content, freshness and tenderness. The first four variables correlate with marginal effects greater than $20 \%$. The $14.4 \%$ and $19.1 \%$ of the population manifested a WTP greater than $21 \%$ of the price they currently pay for meat if it has the attributes organic and safety, respectively.
\end{abstract}

- Key Words: Food safety, Organic meat, Willingness to pay, Contingent valuation, Consumer preferences.

Recibido 22/02/2017.

Aceptado 26/06/2017.

\title{
N Introducción
}

La producción convencional de carne de bovino presenta dificultades para mejorar su rentabilidad, especialmente la que realizan productores pequeños y medianos, quienes obtienen tasas de rentabilidad negativas o muy bajas ${ }^{(1,2)}$. Un factor que afecta la rentabilidad de la ganadería es la disminución del consumo per cápita en México; en 2002 fue 18.4 kg por persona por año, para tener un descenso continuo, y en 2015 se ubicó en $15 \mathrm{~kg}^{(3,4)}$. Una 
variable correlacionada con el consumo es el precio de la carne en canal y al consumidor final, ambas tuvieron una tendencia positiva y creciente, a una tasa anual de 7.3 y $9 \%$ respectivamente en el periodo 2000-2015 $5^{(3,4)}$. La baja en el consumo es explicada por episodios de falta de inocuidad en la carne, precios crecientes y reducción de poder adquisitivo, principalmente ${ }^{(5)}$.

Diversos estudios, tanto en países desarrollados como en desarrollo, documentaron que la producción de productos orgánicos e inocuos pueden ser una alternativa para mejorar la ganancia económica de los productores ${ }^{(6)}$. Sobre este aspecto, las características no sensoriales de los alimentos son cada vez más importantes para los consumidores de estratos socioeconómicos diversos, tales como los atributos orgánico e inocuidad ${ }^{(7)}$; además, para el caso de la carne, toman en cuenta atributos intrínsecos al producto, como el color, la suavidad, y el contenido en grasa; y otros de naturaleza económica como el precio ${ }^{(8)}$.

Esta investigación se enmarca en la teoría de la utilidad aleatoria. Desde un punto de vista económico, el modelo de utilidad aleatoria (MUA) plantea que, dado un segmento homogéneo de la población $\mathrm{N}$ y un conjunto $A_{n}$ de alternativas disponibles para el individuo, una elección es consistente con el MUA si existe un valor de la utilidad $U_{n i} \forall n \in N$ asociado a la alternativa i $\forall i \in A_{n}$, tal que: $P(i)=P\left(U_{n i}>U_{n j}, \forall j \in N A_{n}\right) \quad \forall i \in A_{n}$. La definición anterior supone que la alternativa escogida es la que maximiza la utilidad del individuo ${ }^{(9)}$. De esta forma, la probabilidad de que el individuo n elija la alternativa $i$, corresponde a la probabilidad de que la utilidad de esta alternativa sea mayor que la asociada a cualquiera de las restantes (j), todas ellas pertenecientes al conjunto de alternativas disponibles para el individuo $A_{n}$.

Específicamente, en el análisis de la disponibilidad a pagar por sobreprecio (DAP), una forma de interpretar las elecciones de compra, es utilizando el MUA ${ }^{(10-12)}$. En éste, la función de utilidad está compuesta por un componente determinista y uno aleatorio. El primero refleja factores observables (atributos) que influyen en el nivel de utilidad de elegir el i-ésimo producto. El segundo representa factores no observables, tales como variaciones en las preferencias, comportamiento individual aleatorio y error de medición; la función de utilidad se expresa como $U_{i}=X_{i}^{\prime} \beta+\varepsilon_{i}$, donde ${ }^{{ }_{i}}$ es la utilidad de las i-ésimas alternativas, ${ }_{i}^{\prime} \beta_{\text {i. El }}$ componente determinista $X_{i}$ es un vector de factores observables que influyen en la utilidad, y $\beta$ es un vector de parámetros, que expresan el efecto de las variables independientes sobre la dependiente y es el componente aleatorio ${ }^{(10)}$.

Dado que la utilidad en MUA depende de componentes determinísticos y aleatorios, el cambio en la utilidad, asociado con un cambio en los atributos seleccionados, por ejemplo, inocuidad, orgánica, contenido de grasa, suavidad, y color, entre otros, será igual al cambio 
en los componentes determinísticos y aleatorios, y por lo tanto, la DAP depende del cambio en estos componentes.

La DAP puede ser reescrita, como $D A P=X^{\prime}+{ }_{\text {donde }} X=X_{i} X_{j}={ }_{i}{ }_{j}$.

Para predecir la probabilidad de que la DAP se encuentre en un determinado intervalo, se utilizó la relación entre DAP y los factores que la afectan, por lo que la probabilidad de tener una DAP entre dos niveles es:

$$
\operatorname{Pr}\left(D A P_{\text {bajo }}<D A P \quad D A P_{\text {alto }}\right)=\operatorname{Pr}\left(X^{\prime}+Y_{\text {alto }}\right) \operatorname{Pr}\left(X^{\prime}+<Y_{\text {bajo }}\right)
$$

Donde, $\operatorname{Pr}(\cdot)$ es el operador de probabilidad, $D A P_{\text {bajo y }} D A P_{\text {alto son los límites inferior y }}$ superior de la DAP, y $Y_{\text {alto y }} Y_{\text {bajo }}$ son cambios de umbral en la utilidad ${ }^{(12)}$.

De acuerdo a lo anterior, el objetivo de la investigación es estimar la DAP por parte de los consumidores, porque la carne que consumen sea orgánica e inocua, y determinar las variables que explican el comportamiento de la DAP. Por tanto, la hipótesis de esta investigación es que los consumidores muestran DAP un sobreprecio por los atributos orgánico e inocuidad, y que la magnitud de sobreprecio está explicada por el ingreso, precio, gustos y preferencias de los consumidores, y características sociodemográficas como edad, género, y escolaridad.

\section{Material y métodos}

La metodología general consistió de tres etapas; la primera fue la selección y descripción del enfoque conceptual, el MVC, que define que cuando un bien no tiene un mercado desarrollado, las decisiones de compra de los consumidores no se pueden observar. Para conocer la DAP de los consumidores, bajo las condiciones descritas, el MVC es utilizado con buenos resultados ${ }^{(6,13)}$, ya que puede aproximar mediciones del valor económico debido a que incorpora el valor de uso y el valor de opción de un bien ${ }^{(10)}$, por lo que puede usarse para medir el valor de bienes potenciales (futuros) o hipotéticos (pero realistas), como es el caso de productos con atributos nuevos y no observables. En el caso de los atributos orgánico e inocuidad, lo que los consumidores pagarían es una opción de reducción de riesgos para su salud $^{(14)}$. 
La segunda etapa fue el diseño de muestreo y la aplicación de una entrevista estructurada a 225 consumidores de carne de bovino en seis ciudades del estado de Puebla. El diseño de muestreo fue por conglomerados en dos etapas. Las unidades primarias se seleccionaron por conveniencia: las seis ciudades con mayor población en el estado de Puebla (ciudad de Puebla, Tehuacán, Teziutlán, Cholula, Atlixco, y San Martin Texmelucan) que concentran 653,235 hogares, y representa el $45 \%$ del total del estado. Las unidades secundarias fueron los hogares dentro de cada ciudad. El tamaño de muestra de hogares se determinó por muestreo aleatorio simple ${ }^{(15)}$. Se utilizó un valor de $\mathrm{Z}$ de 1.96 , una precisión del $10 \%$ del valor de la media del ingreso mensual de los hogares en las ciudades muestreadas ${ }^{(16)}$. Cada ciudad se dividió en cuatro cuadrantes, y en cada uno se asignó el $25 \%$ del total de entrevistas predeterminadas para cada ciudad. Las personas entrevistadas se abordaron en carnicerías, centros comerciales y mercados locales. Los encuestadores fueron profesionistas de la Facultad de Economía de la universidad local, capacitados en el MVC y en aplicación de cuestionarios.

El cuestionario se estructuró en cuatro apartados ${ }^{(6,10)}$, a saber: una introducción en la que se explicó la finalidad de la entrevista y se describió el contexto (situación e implicaciones de la producción y consumo de carne convencional versus adicionada con los atributos bajo estudio), como lo indica el $\mathrm{MVC}^{(17)}$; la segunda sección presentó el escenario; la situación de riesgo del consumo de carne convencional versus orgánica e inocua en caso de no valorar adecuadamente sus atributos. En esta sección, y de forma similar a la entrevista a consumidores $^{(13)}$, se incluyeron preguntas para cuantificar la percepción del riesgo para la salud, de consumir carne con clembuterol, hormonas, contenido de colesterol y grasa. Específicamente, se les preguntó que indicaran el nivel de riesgo percibido de cada una de estas cuatro fuentes; ningún riesgo, bajo, moderado, y alto. En términos de puntajes, la preocupación de alto riesgo recibió 3 puntos, preocupación moderada 2, baja preocupación 1 y no preocupación 0. Para resumir los tipos de riesgo individuales, se calculó una variable general de riesgo a la salud (percepción de riesgo) sumando los puntajes de cada una de las cuatro preguntas, y otra que incluyó sólo clembuterol y otra para hormonas. La tercera sección abordó el conocimiento de las preferencias de los consumidores, donde se preguntó por la máxima DAP por el atributo mencionado. La última sección abordó las características sociodemográficas de los encuestados. La pregunta de DAP fue; “¿podría usted señalar, en la tabla que le muestro, en qué rango se encontraría la cantidad adicional máxima que usted pagaría por carne de res orgánica/inocua? Las variables consideradas en el presente estudio se presentan en el Cuadro 1.

La tercera etapa fue el análisis de datos con métodos econométricos. La DAP toma la forma de una variable de respuesta ordenada múltiple, por lo que la mejor elección para la estimación es un modelo econométrico de respuesta ordenada. En este caso, el modelo de DAP utiliza una variable latente de la siguiente manera: $D A P *=X^{\prime} \beta+\varepsilon$, donde DAP* es la DAP latente del consumidor, $X$ es un vector de variables que influyen en la DAP, $\beta$ es un 
vector de parámetros que expresan la relación entre la DAP y X, y $\varepsilon$ es un término de error $\varepsilon$ $\sim$ iid $(0,1)$. Si la DAP* del consumidor cae dentro de cierto rango, su DAP toma un valor numérico que refleja la categoría en la cual está su DAP no observada. En particular, si $\gamma^{j 1<D A P^{*}} \gamma^{j 1}$, entonces $D A P=j 1$ para toda $j=1, \ldots ., J$. Donde $\mathrm{j}$ es la categoría de DAP seleccionada por el consumidor y $\gamma$ es un parámetro categórico. La probabilidad de que la DAP esté en una de las categorías puede escribirse como:

$\operatorname{Pr}(\mathrm{DAP}=\mathrm{j}-1)=\Phi\left(\gamma_{\mathrm{j}}-\mathrm{X}^{\prime} \beta\right)-\Phi\left(\gamma_{\mathrm{j}-1}-\mathrm{X}^{\prime} \beta\right) \forall \mathrm{j} \in \mathrm{J}$

Donde $\Phi(\cdot)$ es una función de densidad acumulativa (CDF), que mide la probabilidad de que la DAP sea menor que el nivel umbral respectivo. Si $\Phi(\cdot)$ es la densidad normal estándar, el modelo de probabilidad es el Probit ordenado ${ }^{(18)}$, el cual permite el cálculo de las probabilidades predichas para cada categoría de DAP y sus efectos marginales. El modelo Probit, al igual que el Logit, tiene una distribución simétrica en forma de campana. La distribución logística es similar a la normal excepto en las colas, que son más pesadas. Por lo tanto, para los valores intermedios de $x$ las dos distribuciones tienden a generar probabilidades similares; entonces, en aplicaciones empíricas, la elección de uno u otro no hace diferencia ${ }^{(19)}$.

El efecto marginal significa que un cambio de una unidad en la variable explicativa dará lugar a un aumento o disminución en la probabilidad prevista igual al tamaño del efecto marginal ${ }^{(20)}$. La especificación del modelo empírico con el que se estimó la DAP por carne orgánica e inocuidad, se describe en la ecuación (2).

$$
\begin{aligned}
& D A P_{i j}={ }_{0}+{ }_{1} \text { Edad }+{ }_{2} \text { Género }+{ }_{3} \text { Escolaridad }+4_{4} \text { Integrantes }+{ }_{5} \text { Cantidad }+{ }_{6} \text { Ingreso }+{ }_{7} \text { Precio } \\
& +{ }_{8} \text { Riesgo+ }{ }_{9} \text { Grasa }+{ }_{10} \text { Frescura }+{ }_{11} \text { Suavidad }+{ }_{12} \text { Color }+
\end{aligned}
$$

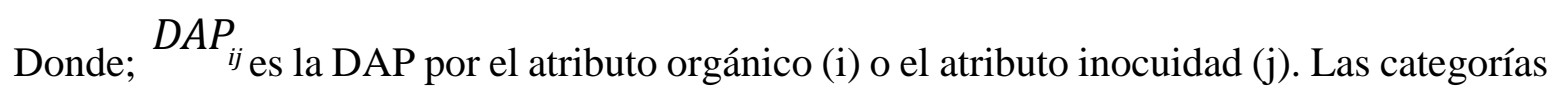
son; $\mathrm{DAP}=0 ; \mathrm{DAP}=1$ (5 \% o menos); $\mathrm{DAP}=2$ (entre 5 y $10 \%)$; DAP=3 (entre 11 y $15 \%$ ); $\mathrm{DAP}=4$ (16 y $20 \%)$; DAP=5 (21\% o mayor). Los rangos de DAP se definieron de acuerdo a estudios previos ${ }^{(6,13)}$. Edad, es la edad del jefe(a) del hogar; Género, es si el jefe(a) es hombre o mujer; Escolaridad, son los años de escolaridad; Integrantes, es el número de integrantes del hogar; Cantidad, son los kilogramos de carne de bovino que consume la familia, por semana; Ingreso, es el intervalo de ingreso del hogar; Precio, es el precio de mercado (pesos por kilogramo); Riesgo, es el nivel de riesgo calculado para cada entrevistado; Grasa, Frescura, Suavidad, y Color se refieren al nivel de importancia por cada uno de estos atributos, medidas en escala Likert de cinco intervalos; $\varepsilon$ es el error del modelo. La descripción de cada variable se detalla en el Cuadro 1. En éste se observa que las variables 
edad y escolaridad se categorizaron, debido a que esto permite descomponer su posible efecto e identificar de forma precisa su contribución y poder explicativo.

\section{Cuadro 1. Variables utilizadas en el modelo de DAP por carne orgánica e inocua}

\begin{tabular}{|c|c|}
\hline Nombre de la variable & Definición de la variable \\
\hline Género & Género del(a) entrevistado; hombre $=1 ;$ mujer $=0$ \\
\hline Edad & Edad del entrevistado, en años \\
\hline Escolaridad & Años de escolaridad sin incluir pre-escolar \\
\hline Integrantes & Número de personas en el hogar \\
\hline Cantidad & Cantidad de carne $(\mathrm{kg})$ consumida por semana en el hogar \\
\hline Ingreso & $\begin{array}{l}\text { Categorías de ingreso del hogar (\$): suma del ingreso de los diferentes } \\
\text { integrantes del hogar, mensual. }\end{array}$ \\
\hline Categorías de ingreso & $\begin{array}{l}\text { Menos de } \$ 5,000 \text {; de } \$ 5,000 \text { a } \$ 9,999 ; \text { de } \$ 10,000 \text { a } \$ 14,999 ; 15,000 \text { y } \\
\text { mayor. La variable ingreso se preguntó por intervalos; la base fue } \\
\$ 5,000 \text {, que es el valor de la canasta alimentaria para una familia } \\
\text { urbana de } 4 \text { integrantes. }\end{array}$ \\
\hline Frecuencia de consumo & $\begin{array}{l}\text { Veces que consume carne de res: diario; cada tercer día; semanal; } \\
\text { quincenal; mensual o mayor. }\end{array}$ \\
\hline Lugar de compra & Supermercado; mercado local o tianguis; carnicería \\
\hline DAP por carne orgánica & $\begin{array}{l}\text { Frecuencia de DAP positiva por carne orgánica; Si tiene } \mathrm{DAP}=1 \text {; No } \\
\text { tiene } \mathrm{DAP}=0\end{array}$ \\
\hline DAP por carne inocua & $\begin{array}{l}\text { Frecuencia de DAP positiva por carne con inocuidad: Si tiene } \mathrm{DAP}=1 \text {; } \\
\text { No tiene DAP }=0\end{array}$ \\
\hline Categorías de DAP por carne orgánica & $\begin{array}{l}\text { Sin DAP; } 5 \% \text { o menos; entre } 5 \text { y } 10 \% \text {; entre } 11 \text { y } 15 \% \text {; entre } 16 \text { y } \\
20 \% ; 21 \text { y } 25 \% \text {; mayor de } 25 \%\end{array}$ \\
\hline $\begin{array}{l}\text { Categorías de DAP por carne con } \\
\text { inocuidad }\end{array}$ & $\begin{array}{l}\text { Sin DAP; } 5 \% \text { o menos; entre } 5 \text { y } 10 \% \text {; entre } 11 \text { y } 15 \% \text {; entre } 16 \text { y } \\
20 \% ; 21 \% \text { a } 25 \% \text { y mayor de } 25 \%\end{array}$ \\
\hline $\begin{array}{l}\text { Importancia; del precio, inocuidad, } \\
\text { orgánico, suavidad, contenido bajo en } \\
\text { grasa, frescura, color rojo }\end{array}$ & $\begin{array}{l}\text { Nivel de importancia de cada atributo; muy baja; baja; moderada; alta; } \\
\text { muy alta }\end{array}$ \\
\hline $\begin{array}{l}\text { Percepción de riesgo; clembuterol, } \\
\text { hormonas, grasas saturadas, y colesterol }\end{array}$ & $\begin{array}{l}\text { Percepción del nivel de riesgo para la salud por presencia en la carne } \\
\text { de sustancias dañinas; nulo, bajo, medio, alto }\end{array}$ \\
\hline $\begin{array}{l}\text { Percepción de contaminación; } \\
\text { supermercado, carnicería, tianguis o } \\
\text { mercado local, tiendas especializadas }\end{array}$ & $\begin{array}{l}\text { Grado de contaminación de la carne de res en diferentes tipos de } \\
\text { expendios: no contaminada; poco contaminada; regular contaminada; } \\
\text { muy contaminada }\end{array}$ \\
\hline $\begin{array}{l}\text { Nivel de credibilidad; médicos } \\
\text { particulares, médicos del sector salud, } \\
\text { SAGARPA, supermercados. }\end{array}$ & $\begin{array}{l}\text { Nivel de credibilidad en la información sobre la inocuidad de la carne } \\
\text { de res; muy baja, baja, moderada, alta, muy alta }\end{array}$ \\
\hline $\begin{array}{l}\text { Nivel de responsabilidad; ganadero, } \\
\text { rastro, supermercado, gobierno }\end{array}$ & $\begin{array}{l}\text { Nivel de responsabilidad en garantizar la inocuidad de la carne; muy } \\
\text { baja, baja, moderada, alta, muy alta }\end{array}$ \\
\hline
\end{tabular}




\section{$\|$ Resultados $\|$}

Las características sociodemográficas de los consumidores son importantes para conocer sus posibles preferencias por carne de bovino. De la muestra de entrevistados, $81.4 \%$ fueron mujeres y el resto hombres, lo que da cuenta de la importancia de las mujeres en las compras del hogar. Al respecto, la revista Merca 2.0 $0^{(21)}$ afirmó que en Chile y Argentina la mujer toma las decisiones de compra de alimentos en $71 \%$ de los hogares, en tanto que en México, la empresa de publicidad Grey, señaló que "las mujeres están detrás del $70 \%$ de las decisiones de compra". La edad de los entrevistados fue de 39.6 años (desviación estándar, ds=11.89), con una escolaridad de 9 años $(\mathrm{ds}=3.45)$. Sobre el particular, la escolaridad promedio en México para hombres y mujeres fue de 9.3 y 9.0 años, en tanto que en Puebla fue de 8.7 y 8.4 respectivamente ${ }^{(16)}$. Los hogares estuvieron integrados por 3.7 integrantes en promedio, con ingreso de 7,600 pesos mensuales $(\mathrm{ds}=1,895)$. La frecuencia de consumo de carne en México está muy arraigada en todos los estratos socioeconómicos; $97.8 \%$ de los entrevistados manifestó consumir carne de res, al menos en ocasiones especiales. La mayor frecuencia de consumo fue semanal $(29.3 \%)$, seguida de quincenal $(27.6 \%)$, cada tercer día $(21.8 \%)$, y ocasiones especiales $(16.9 \%)$. El consumo diario lo realizó el $4.4 \%$ de la muestra.

En México, la cantidad consumida de carne de res ha sido cambiante debido a factores económicos y sociodemográficos, entre ellos, el precio de la carne, el precio de otras carnes, el ingreso de los consumidores, y gustos y preferencias, entre los que se incluye la percepción de riesgos a la salud ${ }^{(16,22)}$. La tasa de crecimiento del consumo en México fue del $4 \%$ anual en el período de 1995-2002 (pasó de 14 a 18.4 kg per cápita), sin embargo, a partir de 2003 inició un descenso, con una tasa de crecimiento de -1.5 de 2003 a 2015, para ubicarse en 15 kg per cápita ${ }^{(3,4)}$. En este estudio, los consumidores que disminuyeron su consumo de carne en los últimos cinco años fue de $46.7 \%$, los que la aumentaron fue $6.67 \%$, y el resto sin cambios. De los que disminuyeron, $81.9 \%$ fueron mujeres y $80 \%$ fueron del estrato de ingreso bajo.

Los datos de la encuesta revelaron que la cantidad consumida presenta variaciones por género, edad, escolaridad, y nivel de ingreso. El promedio general fue de $19.4 \mathrm{~kg} / \mathrm{año}$, con un coeficiente de variación de 0.205 . Los hombres consumieron $18.9 \mathrm{~kg}$ y las mujeres 16.7 $\mathrm{kg}$, sin embargo la diferencia no fue significativa. Para escolaridad, no existieron diferencias 
significativas en la cantidad consumida, para los estratos de 6 años (12.8 kg), 7-9 años (13.8 $\mathrm{kg}$ ), y 10 a 12 años $(18.6 \mathrm{~kg}$ ); sin embargo se encontraron diferencias para el estrato de consumidores con 13 o más años de escolaridad, quienes consumieron $26.3 \mathrm{~kg}$. El estrato de ingreso bajo tiene un consumo per cápita de $12.1 \mathrm{~kg}$, los de ingreso medio $23.9 \mathrm{~kg}$ y los de alto $30.7 \mathrm{~kg}$.

Respecto al lugar de compra de la carne, el $44 \%$ la realizó en carnicerías, el $30 \%$ en mercados locales o tianguis y el $26 \%$ en supermercados y tiendas especializadas en carne. Los consumidores con DAP sobreprecio por carne orgánica fue del $66.7 \%$ y porque sea inocua del $76.4 \%$. En una escala de cinco intervalos, en orden jerárquico, los consumidores consideraron como importante y muy importante la inocuidad (86.8\%), orgánica (71.2\%), contenido de grasa $(69.9 \%)$, suavidad $(66.6 \%)$, precio $(56.9 \%)$, y el color de la carne (56.4 $\%)$. Estos datos pueden ser un insumo importante en una estrategia de promoción del consumo de carne.

La importancia de conocer la percepción de riesgo para la salud por parte de los consumidores, sobre la inocuidad de la carne, radica en que permite una mejor comprensión de los factores que determinan la demanda y para mejorar el diseño de estrategias de la industria para contrarrestar movimientos negativos de la demanda ${ }^{(23)}$. Los consumidores percibieron que la carne está contaminada $(80 \%)$ en algún grado con sustancias o microorganismos que pueden dañar la salud. El índice general de percepción de contaminación fue de 0.61 , en escala de 0 a 1 . Entre los más mencionados estuvieron el clembuterol y las hormonas, sin embargo también se mencionó como riesgo para la salud la presencia de colesterol y grasas. Los lugares de compra donde se percibió que la carne puede tener mayores cantidades de contaminantes son los mercados locales y tianguis, y carnicerías. El índice general de contaminación fue del 0.49, en escala de 0 a 1 (Cuadro 2).

La información sobre la inocuidad de la carne de res, tiene importancia en la medida que genera confianza en los consumidores, lo que se traduce en una mayor demanda ${ }^{(22)}$. Los entrevistados manifestaron credibilidad alta y muy alta, si la información la reciben del sector salud $(74.4 \%)$, de médicos privados $(70.2 \%)$, supermercados $(56.5 \%)$, y SAGARPA $(53.4 \%)$. Sobre las instancias que deberían garantizar la inocuidad, en opinión del $67.24 \%$ de los consumidores, el gobierno tiene un nivel de responsabilidad alto y muy alto, seguido del ganadero y el rastro, según el 63.8 y $63.3 \%$ respectivamente (Cuadro 2).

Cuadro 2. Percepción de riesgo, grado de contaminación y credibilidad (\%)

\begin{tabular}{lcccc}
\hline Percepción de riesgo & Clembuterol & Hormonas & Grasas & Colesterol \\
\hline Ninguno & 20.0 & 20.0 & 21.8 & 12.9 \\
Bajo & 15.1 & 21.8 & 19.1 & 13.3 \\
Moderado & 24.0 & 28.4 & 27.6 & 28.9 \\
Alto & 40.9 & 29.8 & 31.6 & 44.9 \\
\hline
\end{tabular}




\begin{tabular}{lcccc}
\hline Índice de percepción de riesgo & 0.62 & 0.55 & 0.56 & 0.68 \\
\hline Grado de contaminación & $\begin{array}{c}\text { Mercado local y } \\
\text { tianguis }\end{array}$ & $\begin{array}{c}\text { Súper } \\
\text { mercados }\end{array}$ & Carnicería & $\begin{array}{c}\text { Tiendas } \\
\text { especializadas }\end{array}$ \\
\hline No contaminada & 11.8 & 31.2 & 13.4 & 31.1 \\
Poco contaminada & 17.7 & 46.1 & 12.5 & 51.1 \\
$\begin{array}{l}\text { Regular contaminada } \\
\text { Muy contaminada }\end{array}$ & 29.6 & 17.0 & 33.1 & 13.3 \\
Índice de percepción de & 41.0 & 5.6 & 41.0 & 4.4 \\
contaminación & 0.66 & 0.32 & 0.67 & 0.30 \\
\hline Nivel de credibilidad & Médicos & SAGARPA & Sector salud & Súper \\
& & & & \\
\hline Muy baja & 2.7 & 6.3 & 5.4 & 2.3 \\
Baja & 4 & 13.1 & 12.2 & 11.1 \\
Moderada & 23.1 & 27.1 & 8.1 & 30.2 \\
Alta & 22.4 & 30.2 & 31.4 & 23.4 \\
Muy alta & 47.8 & 23.2 & 43 & 33.1 \\
\hline & & Rastro & Súper & Gobierno \\
Nivel de responsabilidad & Ganadero & & mercados & \\
\hline Muy baja & & 9.86 & 21.81 & 5.38 \\
Baja & 7.6 & 12.26 & 30.45 & 1.88 \\
Moderada & 13.17 & 14.48 & 24.48 & 25.45 \\
Alta & 15.38 & 19.95 & 13.12 & 26.13 \\
Muy alta & 19.05 & 43.44 & 10.13 & 41.11 \\
\hline & 44.8 & & & \\
\hline
\end{tabular}

Sobre la pregunta de DAP por los atributos orgánico e inocuidad, el 33.3 y $23.6 \%$ de los entrevistados no mostró DAP. La mayor parte de los consumidores manifestó una DAP menor al $15 \%$; $43.5 \%$ para el atributo orgánico y $47.6 \%$ para la inocuidad. Se identificó un importante segmento de consumidores, que compran principalmente en supermercados y tiendas especializadas, que tiene una DAP alta; 14.4 y $19.1 \%$ de los consumidores tienen DAP de $21 \%$ o mayor por el atributo orgánico (Cuadro 3).

Cuadro 3. Frecuencia y porcentaje de DAP por los atributos orgánico e inocuidad

\begin{tabular}{lcccc}
\hline \multicolumn{1}{c}{ Categorías de DAP } & $\begin{array}{c}\text { DAP por el atributo "Orgánico" } \\
\text { Frecuencia }\end{array}$ & $\begin{array}{c}\text { DAP por el atributo "Inocuidad" } \\
\text { Porcentaje }\end{array}$ & $\begin{array}{c}\text { Frecuencia } \\
\text { Porcentaje }\end{array}$ \\
\hline Sin DAP & 75 & 33.3 & 50 & 22.6 \\
$5 \%$ o menos & 41 & 18.2 & 46 & 20.4 \\
Entre 5 y $10 \%$ & 32 & 14.2 & 33 & 14.7 \\
Entre 11 y $15 \%$ & 25 & 11.1 & 28 & 12.4 \\
Entre 16 y 20\% & 20 & 8.9 & 25 & 11.1 \\
Entre 21 Y 25\% & 19 & 8.4 & 24 & 10.7 \\
Más del 25 \% & 13 & 5.8 & 19 & 8.4 \\
Total & 225 & 100 & 225 & 100 \\
\hline
\end{tabular}


$\mathrm{DAP}=$ disponibilidad a pagar por sobreprecio.

Las estimaciones de los parámetros y las estadísticas de ajuste del modelo Probit se presentan en el Cuadro 4. La prueba de log-likelihod indica que el modelo estimado tiene poder explicativo satisfactorio; $1 \mathrm{~J} \mathrm{Ji}^{2}$ fue significativa a un nivel de probabilidad del $5 \%$, por lo que la hipótesis nula de que los coeficientes estimados son igual a cero se rechazó al nivel del $5 \%$. Seis variables en el modelo explicativo de DAP por carne orgánica y cinco para el de DAP por inocuidad fueron significativas $(P<0.05)$. La edad resultó significativa únicamente en el estrato de 35 a 59 años, la escolaridad en el estrato de 13 años o mayor, el ingreso en los estratos de 10 a 15 mil, y en el de "mayor de 15 mil, la variable "percepción de riesgo" para el caso del modelo por inocuidad, y el contenido bajo en grasa.

Cuadro 4. Resultados del modelo Probit de la DAP por carne orgánica e inocuidad

\begin{tabular}{|c|c|c|c|c|c|c|}
\hline \multirow[b]{2}{*}{ Variables explicativas } & \multicolumn{3}{|c|}{ Modelo DAP por carne orgánica } & \multicolumn{3}{|c|}{$\begin{array}{l}\text { Modelo DAP por carne con } \\
\text { inocuidad }\end{array}$} \\
\hline & $\boldsymbol{\beta}$ & $\begin{array}{c}\text { Error } \\
\text { estándar }\end{array}$ & Valor de $\mathbf{t}$ & $\boldsymbol{\beta}$ & $\begin{array}{c}\text { Error } \\
\text { estándar }\end{array}$ & $\begin{array}{c}\text { Valor de } \\
\text { t }\end{array}$ \\
\hline Edad; 35 años o menos & 0.137 & 0.2210 & 0.62 & -0.145 & 0.2164 & -0.67 \\
\hline Edad; entre 35 y 59 años & -0.036 & 0.0167 & $-2.15 * *$ & -0.126 & 0.0492 & $-2.56 * *$ \\
\hline Edad; 60 años o más & 0.744 & 0.4482 & 1.66 & -0.016 & 0.8000 & -0.02 \\
\hline Género & -0.129 & -0.2867 & 0.45 & -0.281 & -0.2782 & 1.01 \\
\hline Escolaridad; 7-9 años & -0.327 & 0.2137 & -1.53 & -0.033 & 0.2063 & -0.16 \\
\hline Escolaridad; 10-12 años & -0.559 & 0.4267 & -1.31 & -0.123 & 0.2365 & -0.52 \\
\hline Escolaridad;13 años o más & 1.029 & 0.3476 & $2.96 * *$ & 0.673 & 0.3399 & $1.98 * *$ \\
\hline Integrantes & -0.027 & 0.0600 & -0.45 & -0.029 & 0.0569 & -0.51 \\
\hline Cantidad & 0.009 & 0.0094 & 0.96 & 0.011 & 0.2200 & 0.05 \\
\hline Ingreso; menos de 5,000 & -0.225 & 0.1991 & -1.13 & -0.286 & 0.1932 & -1.48 \\
\hline Ingreso; 5,000 a 9,999 & -0.708 & 0.6378 & -1.11 & -0.234 & 0.3391 & -0.69 \\
\hline Ingreso; 10,000 a 14,999 & 1.58 & 0.3583 & $4.41 * *$ & 0.964 & 0.3406 & $2.83 * *$ \\
\hline Ingreso; 15,000 y mayor & 1.615 & 0.4228 & $3.82 * *$ & 0.882 & 0.1938 & $4.55 * *$ \\
\hline importancia del precio & -0.197 & 0.1187 & -1.66 & -0.128 & 0.2510 & -0.51 \\
\hline Percepción de riesgo & 0.096 & 0.0410 & $2.34 * *$ & 0.35 & 0.1264 & $2.77 * *$ \\
\hline Contenido de grasa & 0.234 & 0.0857 & $2.73 * *$ & 0.1946 & 0.0821 & $2.37 * *$ \\
\hline Frescura & 2.201 & 0.7777 & $2.83 * *$ & 0.5094 & 1.2424 & 0.41 \\
\hline Suavidad & 0.025 & 0.0110 & $2.28 * *$ & -0.5746 & 0.3380 & -1.7 \\
\hline Color (rojo cereza) & -0.332 & 0.2441 & -1.36 & -0.1941 & 0.1377 & -1.41 \\
\hline -2Log likelihood & \multicolumn{3}{|c|}{$-259.91507 * *$} & \multicolumn{3}{|c|}{$-275.02843 * *$} \\
\hline Seudo R2y R2 escalado & \multicolumn{3}{|c|}{$0.1472 / 0.39$} & \multicolumn{3}{|c|}{$0.177 / 0.41$} \\
\hline Prueba LR $\left(\mathrm{Ji}^{2}\right)$ & \multicolumn{3}{|c|}{$189.71 * *$} & \multicolumn{3}{|c|}{$186.23 * *$} \\
\hline
\end{tabular}

$\mathrm{DAP}=$ disponibilidad a pagar por sobreprecio.

**Indica significancia al $95 \%$.

Nota 1: En los dos modelos, la variable dependiente es la DAP. 
Nota 2: $\beta$ son los coeficientes del modelo Probit (Ver ecuación 2).

Los coeficientes del modelo están expresados como el logaritmo de la razón de probabilidades (odd ratios). La interpretación es que por cada incremento de una unidad en la variable explicativa, la variable de respuesta (DAP) cambia en alguna de sus categorías, en la magnitud del coeficiente ${ }^{(19)}$. Los coeficientes del modelo proporcionan información útil, sin embargo, por la forma en que están expresados no son muy informativos. Para hacerlos más informativos, se calculó el efecto marginal, que se define como el impacto de un cambio en una variable explicativa sobre las probabilidades predichas de DAP. Para una variable explicativa en particular, un cambio de una unidad impacta en un aumento o disminución en las probabilidades predichas en la magnitud del efecto marginal ${ }^{(19)}$. El Cuadro 5 presenta los efectos marginales de las variables explicativas del modelo Probit, para cada uno de los niveles de DAP. Respecto a la edad, ser menor de 35 años redujo las probabilidades de pagar un sobreprecio mayor al $5 \%$. Pertenecer al estrato de 36 años o más aumentó las probabilidades de DAP mayor al $5 \%$. Los efectos marginales de la variable género, indicaron que las mujeres son más propensas a pagar una prima de $6 \%$ o más. En escolaridad, el estrato de 7-9 años, aumentó las probabilidades, en $10 \%$, de DAP nula. Estar en los estratos de 10 a 12 años y 13 o mayor aumentó las probabilidades de pagar sobreprecios mayores al $6 \%$. Esta última categoría aumentó las probabilidades en $20.1 \%$ de DAP mayor al $10 \%$.

Cuadro 5. Efectos marginales en el modelo de DAP por carne orgánica

\begin{tabular}{lrrrrrr}
\hline Variable & DAP=0 & DAP=1 & DAP=2 & DAP=3 & DAP=4 & DAP=5 \\
\hline Edad; 35 años o menos & 0.0424 & 0.0119 & -0.0159 & -0.0269 & -0.0068 & -0.0048 \\
Edad; 36 a 59 años & -0.0110 & -0.0031 & 0.0041 & 0.0070 & 0.0018 & 0.0013 \\
Edad; 60 años o más & 0.2296 & 0.0647 & -0.0860 & -0.1457 & -0.0366 & -0.0260 \\
Género & -0.0397 & -0.0112 & 0.0148 & 0.0252 & 0.0063 & 0.0045 \\
Escolaridad; 7-9 años & 0.1008 & 0.0284 & -0.0377 & -0.0640 & -0.0160 & -0.0114 \\
Escolaridad; 10-12 años & -0.1725 & -0.0486 & 0.0646 & 0.1095 & 0.0275 & 0.0195 \\
Escolaridad; 13 años y más & -0.3174 & -0.0894 & 0.1189 & 0.2014 & 0.0505 & 0.0359 \\
Integrantes & -0.0083 & -0.0023 & 0.0031 & 0.0053 & 0.0013 & 0.0009 \\
Ingreso; de 5-10 mil & 0.0693 & 0.0195 & -0.0260 & -0.0440 & -0.0110 & -0.0079 \\
Ingreso; de 11-15 mil & -0.2185 & -0.0615 & 0.0818 & 0.1386 & 0.0348 & 0.0247 \\
Ingreso; mayor a 15 mil & -0.4874 & -0.1372 & 0.1825 & 0.3093 & 0.0776 & 0.0552 \\
importancia del precio & 0.0299 & 0.0084 & -0.0112 & -0.0190 & -0.0048 & -0.0034 \\
Percepción de riesgo & -0.3073 & -0.0865 & 0.1151 & 0.1950 & 0.0489 & 0.0348 \\
Contenido de grasa & -0.0564 & -0.0923 & -0.1025 & 0.0922 & 0.2025 & 0.1522 \\
Frescura & 0.6792 & 0.1912 & -0.2544 & -0.4310 & -0.1081 & -0.0769 \\
Suavidad & 0.0078 & 0.0022 & -0.0029 & -0.0050 & -0.0012 & -0.0009 \\
Color & 0.1026 & 0.0289 & -0.0384 & -0.0651 & -0.0163 & -0.0116 \\
\hline
\end{tabular}


$\mathrm{DAP}=$ disponibilidad a pagar sobreprecio.

El ingreso es una variable que ha sido citada en la literatura como importante en explicar la DAP por atributos de calidad en carne de res. En comparación con el estrato más bajo de ingreso (menos de 5 mil), se encontró que estar en el estrato de 5-10 mil aumentó la probabilidad de DAP de $5 \%$ o menos. Estar en el estrato más alto (15 mil y más) aumentó las probabilidades en $30.9 \%$ de DAP sobreprecios entre $10-15 \%$.

La percepción de riesgo, construida para conocer el riesgo percibido por el consumo de carne de res, indicó la presencia de aversión al riesgo (a mayor percepción de riesgo, mayor DAP). Esta variable indujo que las probabilidades de pagar sobreprecios 10-15\% aumentaron en $19.5 \%$, y pagar entre $15-20 \%$ aumentaron en $4.9 \%$.

En general, el sentido y dirección de los efectos marginales, en el modelo de DAP por inocuidad fueron similares a los del modelo de DAP por carne orgánica (Cuadro 6), sin embargo existieron algunas diferencias. Respecto a la edad, ser menor de 35 años aumentó las probabilidades de pagar un sobreprecio igual o mayor del $5 \%$ y redujo en $3.9 \%$ la probabilidad de cero sobreprecio. La dirección de los efectos de ser mujer, es la misma que en el modelo de DAP por orgánico, pero los cambios fueron más grandes. La escolaridad, las probabilidades de pagar sobreprecios mayores al $5 \%$ aumentaron.

Cuadro 6. Efectos marginales en el modelo de DAP por el atributo inocuidad

\begin{tabular}{lcccccc}
\hline \multicolumn{1}{c}{ Variable } & DAP=0 & DAP=1 & DAP=2 & DAP=3 & DAP=4 & DAP=5 \\
\hline Edad; 35 años o menos & -0.0398 & -0.0179 & 0.0176 & 0.0233 & 0.0042 & 0.0126 \\
Edad; 36 a 59 años & -0.0346 & -0.0156 & 0.0153 & 0.0203 & 0.0036 & 0.0110 \\
Edad; 60 años o más & 0.0018 & 0.0008 & -0.0008 & -0.0011 & -0.0002 & -0.0006 \\
Género & -0.0772 & -0.0348 & 0.0341 & 0.0453 & 0.0081 & 0.0245 \\
Escolaridad; 7-9 años & -0.0092 & -0.0041 & 0.0041 & 0.0054 & 0.0010 & 0.0029 \\
Escolaridad; 10-12 años & -0.0337 & -0.0152 & 0.0149 & 0.0198 & 0.0035 & 0.0107 \\
Escolaridad; 13 o más & -0.1847 & -0.0832 & 0.0816 & 0.1083 & 0.0194 & 0.0586 \\
Integrantes & 0.0081 & 0.0037 & -0.0036 & -0.0048 & -0.0009 & -0.0026 \\
Ingreso; de 5-10 mil & 0.0785 & 0.0354 & -0.0347 & -0.0460 & -0.0082 & -0.0249 \\
Ingreso; de 11-15 mil & -0.0643 & -0.0290 & 0.0284 & 0.0377 & 0.0067 & 0.0204 \\
Ingreso; 15 mil o mayor & -0.2643 & -0.1190 & 0.1168 & 0.1550 & 0.0277 & 0.0838 \\
Importancia del precio & 0.0077 & 0.0035 & -0.0034 & -0.0045 & -0.0008 & -0.0025 \\
Percepción de riesgo & -0.0224 & -0.0962 & 0.0945 & 0.1253 & 0.2137 & 0.0677 \\
Contenido de grasa & -0.0322 & -0.0823 & -0.1088 & 0.1161 & 0.1544 & 0.1521 \\
Frescura & -0.1396 & -0.0629 & 0.0617 & 0.0819 & 0.0146 & 0.0443 \\
Suavidad & 0.1575 & 0.0710 & -0.0696 & -0.0924 & -0.0165 & -0.0499 \\
\hline
\end{tabular}




\begin{tabular}{lrrrrrr}
\hline Color & 0.0532 & 0.0240 & -0.0235 & -0.0312 & -0.0056 & -0.0169 \\
\hline DAP= disponibilidad a pagar por sobreprecio. & & & & &
\end{tabular}

A diferencia del modelo de DAP por lo orgánico, en este caso, solo dos categorías de ingreso (11-15 mil y 15 mil o mayor) mostraron DAP sobreprecios mayores al $5 \%$. La percepción de riesgo indica que los consumidores que mostraron niveles altos del índice de percepción de riesgo, mostraron DAP sobreprecios, ligeramente más altos; el cambio en las probabilidades de pagar sobreprecios entre $15-20 \%$, fue de $21.4 \%$

\section{Discusión}

En esta investigación se encontró que la DAP un sobreprecio, en promedio, por los atributos estudiados, fue de $12.1 \%$ para lo orgánico y $13 \%$ para la inocuidad. Sin embargo, dos aspectos son igualmente relevantes: el $33.3 \%$ y $22.6 \%$ de los entrevistados mostraron DAP de cero para los atributos orgánico e inocuidad, respectivamente. Estos valores son similares a reportes en estudios previos ${ }^{(24)}$. Por otro lado, se encontró que 14.2 y $19.1 \%$ reportaron una DAP igual o mayor al $21 \%$. Estos valores muestran el potencial de estos atributos para agregar valor a la producción, y aportan evidencia de que estos atributos mejoran la utilidad de un importante subconjunto de consumidores. Estos resultados no son muy diferentes a lo reportado previamente ${ }^{(25)}$ en un estudio en Bangladesh, donde encontraron que $41 \%$ de entrevistados mostraron una DAP de $5 \%$ y que el $30 \%$ una DAP de $10 \%$. En un estudio en Estados Unidos ${ }^{(26)}$ encontraron una DAP del $11.6 \%$ por carne etiquetada como "natural" y en un estudio en Canadá(6) reportaron que el $29 \%$ mostró una DAP de 6-10\% y el $10 \%$ una DAP mayor al $11 \%$. Sin embargo otros autores han encontrado valores más altos de DAP por carne orgánica e inocua ${ }^{(13,27)}$, en un estudio en ciudades Italianas reportaron una DAP promedio del 20 y $30 \%$ respectivamente.

Con respecto a la segunda parte de la hipótesis, los factores explicativos de la DAP que mostraron significancia $(P<0.05)$ fueron la edad, la escolaridad, el ingreso, la percepción de riesgo, importancia del contenido de grasa, y la frescura. La edad fue significativa $(P<0.05)$ sólo en el estrato de 35 a 59 años. Resultados similares fueron reportados por otros ${ }^{(23,6)}$, para el estrato de $\leq 35$ años. Lo anterior implica que, en este estrato, se tiene mayor aversión al riesgo acompañado de mayor ingreso, debido a que estas dos variables están correlacionadas negativa y positivamente con la DAP. El coeficiente de la variable género, indicó una relación 
negativa con DAP, sin embargo, ésta no fue significativa en ninguno de los dos modelos estimados.

La variable escolaridad fue significativa en el estrato de 13 años o más, tanto en el modelo de DAP por el atributo orgánica como el de inocuidad. El nivel educativo estuvo correlacionado significativamente $(P<0.05)$ con ingreso y percepción de riesgo ${ }^{(22)}$. Estas variables podrían usarse en definir un nicho de mercado. En esta dirección, estudios previos $^{(25,13)}$ reportaron significancia estadística de la variable educación.

El ingreso es una variable explicativa de la DAP sobreprecio, en los estratos de 11 a 15 mil y 15 mil y mayor, en ambos modelos. El estrato de mayor ingreso generó efectos marginales de $31 \%$ en el modelo de DAP por el atributo orgánico y de $16 \%$ para el de inocuidad. Esta variable fue encontrada significativa en diferentes investigaciones ${ }^{(28)}$, donde se mostró que a mayor ingreso, la DAP aumenta, con efectos marginales del $13 \%$. De forma similar, otros investigadores $^{(25)}$ encontraron significancia y efectos marginales del $32 \%$. La implicación de lo anterior es que los estratos de ingreso alto, que asciende al $6 \%$ de los entrevistados, representan un importante nicho de mercado.

La variable "percepción de riesgo" en países como México, Estados Unidos, Canadá y Japón ${ }^{(22)}$, ha sido un factor explicativo de la disminución en el consumo per cápita de carne de res. En Canadá y Estados Unidos el $20 \%$ de los entrevistados redujo su consumo en los últimos cuatro años, mientras que en Japón y México lo han hecho el 55 y 30 \%, respectivamente. En este estudio, percepción del riesgo fue significativa en ambos modelos, con efectos marginales de 19.5 y $21 \%$, respectivamente.

La preferencia por carne baja en grasa fue significativa en los dos modelos estimados, probablemente debido a que este atributo está altamente relacionado con el cuidado de la salud de un segmento de la población. La frescura y la suavidad fueron significativos sólo en el modelo de DAP por carne orgánica. Lo anterior, probablemente debido a que los consumidores con DAP por inocuidad, más preocupados por su salud, no tienen en alta estima estos atributos y mostraron baja preferencia por ellos.

La percepción de contaminación es un aspecto que deberá atenderse. El índice calculado de contaminación de 0.61 , fue similar al reportado en estudios previos ${ }^{(22)}$. Una posibilidad es la promoción genérica, acompañada de medidas correctivas de inocuidad en la cadena de producción, y especialmente en los lugares de compra, como mercados locales, carnicerías y supermercados.

Este estudio aportó elementos para mejorar la toma de decisiones de los agentes económicos de la cadena productiva carne de res; la existencia de DAP, el porcentaje de la muestra con DAP, los atributos más preferidos, los factores explicativos de la DAP, las proporciones del lugar de compra, y la percepción de contaminación en esos centros de venta. Queda pendiente, el estudio de las implicaciones en la estructura de costos de la producción de carne 
orgánica y la implantación de sistemas de inocuidad de la carne, de forma creíble para los consumidores.

\section{| Conclusiones e implicaciones |}

Al menos dos terceras partes de los entrevistados pagarían un sobreprecio por la presencia en la carne de los atributos inocuidad y orgánico. Los consumidores son más propensos a pagar un sobreprecio si están en el estrato de edad de 35 a 59 años, con escolaridad de más de 13 años, estrato de ingreso medio y alto, y si son adversos al riesgo (percepción de riesgo alto). Estas variables generaron los efectos marginales más grandes. El porcentaje de la muestra con las características anteriores se estimó en $16 \%$. Lo anterior, podría permitir el diseño de una estrategia de marketing, enfocada a este nicho de mercado. Los esfuerzos de marketing deben encaminarse en identificar el nicho de mercado (los consumidores que se ajusten a este perfil) y a hacer llegar la información de las ventajas de estos atributos para conservar o mejorar la salud de los consumidores, con énfasis en la familia. En esta estrategia, es importante la participación del sector salud, médicos, supermercados, y SAGARPA, para hacer llegar información a todos los consumidores sobre los atributos incluidos.

\section{- Literatura citada}

1. Rebollar-Rebollar A, Hernández-Martínez J, Rebollar-Rebollar S, Guzmán-Soria E, García-Martínez A, González-Razo FJ. Competitividad y rentabilidad de bovinos en corral en el sur del estado de México. Trop Subtrop Agroecosyt 2011;14(2):691-698.

2. Gamboa-Mena JV, Magaña-Magaña MA, Rejón-Ávila M, Martínez PVC. Eficiencia económica de los sistemas de producción de carne bovina en el municipio de Tizimín, Yucatán, México. Trop Subtrop Agroecosyt 2005;5(2):79-84.

3. SNIIM. Sistema Nacional de Información e Integración de Mercados. 2016. Datos de precios de carne bovino. http://www.economia-sniim.gob.mx/2010prueba/Bovinos.asp. Consultado 16 nov, 2016. 
4. CNOG. Confederación Nacional de Organizaciones Ganaderas. 2016. Datos de precios de carne bovino. http://www.cnog.org.mx. Consultado 16 nov, 2016.

5. Norat T, Riboli E. Meat consumption and colorectal cancer: A review of epidemiologic evidence. Nutr Rev 2001:59(2):37-47.

6. Cranfield JAL, Magnusson E. Canadian Consumer's willingness to pay for pesticide free food products: An ordered probit analysist. IFAMR 2003;6(4):13-30.

7. Moser R, Raffaelli R, Thilmany-McFadden D. Consumer preferences for fruit and vegetables with credence-based attributes: A Review. IFAMR 2011;14(2):121-141.

8. Burton S, Andrews JC. Age, product nutrition, and label format effects on consumer perceptions and product evaluations. J Cons Aff 1996;30(1):68-89.

9. Block HD, Marschak J. Random orderings and stochastic theories of responses, In: Olkin S, et al editors. Contributions to probability and statistics. Stanford, Calif: Stanford University Press; 1960:97-132.

10. Bateman IJ, Willis KG. Valuing environmental preferences: theory and practice of the contingent valuation method in the US, EU, and developing countries. 1rst ed. US: Oxford University Press Inc; 2001.

11. Baltas G, Doyle P. Random utility models in marketing research: A survey. J Bus Res 2001;51(2):115-125.

12. Veeman M, Adamowicz W. Consumer's perceptions of enviromental risks and the demand for food safety. AARI project 960730:2000:1-33.

13. Boccaletti S, Nardella M. Consumer willingness to pay for pesticide-free fresh fruit and vegetables in Italy. IFAMR 2000;3(3):297-310.

14. Misra KS, Huang LC, Ott SL. Consumer willingness to pay for pesticide-free fresh produce. Western J Agr Econ 1991;16(2):218-227.

15. Starnes SD, Yates SD, Moore SD. The practice of statistics. 4th ed. New York, USA: WH Freedman and Co; 2008.

16. ENIGH. Encuesta Nacional de Ingresos y Gastos de los Hogares. Bases de datos estadísticos 2014. http://www.beta.inegi.org.mx/ proyectos/enchogares/regulares/enigh/tradicional/2014/. Consultado 16 de Nov de 2016.

17. Carson RT. Contingent valuation: A user's guide. Environ Sci Technol 2000;34(8): 1413-1418. 
18. Long JS, Freese J. Regression models for categorical dependent variables using stata. 2nd ed. Texas, USA: Stata Press; 2006.

19. Green WH. Econometric analysis. Fifth ed. New Jersey, USA: Ed. Prentice Hall; 2002.

20. Willians R. Using the margins command to estimate and interpret adjusted predictions and marginal effects. The Stata J 2012;12(2):308-331.

21. Revista Merca2.0. http://www.merca20.com/tag/decision-de-compra/. Consultado 27 ene, 2016.

22. Schroeder CT, Tonsor TG, Pennings JME, Mintert J. Consumer food safety risk perceptions and attitudes: Impacts on beef consumption across countries. The BE J Econ Anal Pol 2007;7(1):1-27.

23. Tonsor GT, Schroeder TC, Pennings JME. Factors impacting food safety risk perceptions. J Agr Econ 2009;60(3):625-644.

24. Cicia G, Colantuoni F. Willingness to pay for traceable meat attributes: A meta-analysis. Int J Food Sytem Dynamics 2010;(3):252-263.

25. Sarma PK, Raha SK. Consumer's willingness to pay for organic beef: Evidence from Dhaka City. J Bangladesh Agril Univ 2016;14(1):83-91.

26. Curtis K, Feuz S, Aybar N. Consumer willingness to pay for specialty meats. Finance and Economics, Utah State University. 2012.

27. Corsi A, Novelli S. Consumer willingness to pay a price for organic beef meat. $X^{\text {th }}$ EAAE Cong "Exploring Diversity in the European Agri-Food System". Zaragoza, Spain. 2002:28-31.

28. Naanwaab C, Yeboah OA, Kyei FO, Sulakvelidze A, Goktepe I. Evaluation of consumers' perception and willingness to pay for bacteriophage treated fresh produce. Bacteriophage 2014;4(4):e979662. 